\title{
ANTIBINDING OF ATOMIC ELECTRONS IN STRONG INHOMOGENEOUS MAGNETIC FIELDS
}

\author{
D. H. JAKUBASSA-AMUNDSEN
}

\begin{abstract}
The ground-state energy of heavy one-electron ions in an inhomogeneous locally bounded magnetic field is estimated by the variational principle. The ions are described by means of the pseudorelativistic Herbst/Chandrasekhar operator. Two classes of magnetic fields are considered which model a field-free region around the central charge. It is shown that for a certain size of this region the ground-state energy becomes positive and increases strongly with the magnetic field strength. This behaviour is in contrast to the two-dimensional case where electrons can be bound by such a field-free region.
\end{abstract}

\section{INTRODUCTION}

The interest in electrons subject to inhomogeneous magnetic fields was revived by the preparation of graphene monolayers which give rise to a purely twodimensional electronic motion. It was suggested by Egger and coworkers to apply a static magnetic field, oriented perpendicular to the monolayer, which is constant outside a cylinder of radius $r_{0}$ and zero inside (Fig.1a). In a rigorous theoretical approach, based on the two-dimensional Dirac-Weyl equation, it was shown that an electron gets bound in such a field-free disk, the number of bound states increasing with $r_{0}[1,2]$. In accord with the experimental spectrum the electron mass is thereby set equal to zero [1]. Also a mathematical analysis of the two-dimensional confinement by this magnetic field was provided for the case of interacting massless multi-fermions [3].

In the three-dimensional atomic case where the confinement in the $x_{3}$-direction (the field direction) is achieved by a fixed central Coulomb potential, a massless particle can be simulated by an appropriate magnetic field of very large strength. A magnetic field which shows this feature was introduced in [4] and is of asymptotic growth but has also a depleted interior region (Fig.1b). In the presence of such a field the Herbst operator, used to model relativistic atomic systems [5], exhibits a scaling property which reduces the mass term, the more so, the larger the field strength $B$.

In the present work the question is addressed whether for magnetic fields of the type discussed above the binding of the electron increases with the size of the depleted region as in the two-dimensional case. Taken into consideration that the influence of magnetic fields in two and three dimensions is often quite different 
(e.g. the ground-state binding of the atomic electron in a homogeneous magnetic field increases with $B$; see e.g. the review by Lai [6]), the answer is not easily predictable.

Rather than progressing with a fully relativistic approach we estimate the ground-state energy of the electron by means of a more transparent variational calculation. A trial function which is suitable in a wide range of magnetic fields was introduced by Rau and coworkers [7] in the context of Schrödinger operators. It was shown for homogeneous magnetic fields that this trial function not only provides the correct limits for $B \rightarrow 0$ and $B \rightarrow \infty$, but that also for intermediate field strengths it is able to reproduce the binding energies from accurate numerical calculations [8]. Slightly modified trial functions were used in the context of the pseudorelativistic Brown-Ravenhall operator [9, 10] and the Herbst operator [4]. However, restriction was made to the presence of very strong magnetic fields.

The paper is organized as follows. For the two types of magnetic fields discussed above the ground-state energy of the Herbst operator is estimated in Sections 2.1 and 2.2, respectively (using the trial function from [7]), and its dependence on the field parameters $B, \tau$ and $r_{0}$ is investigated for the fixed central charge $Z=80$. In Sections 3 and 4 the stability of these results is tested by choosing different types of trial functions, including such which mimick relativistic effects. A short conclusion is given in Section 5.

\section{VARIATIONAL PRINCIPLE FOR THE HERbST OPERATOR}

Relativistic one-electron ions are conventionally described by the Dirac operator $H$ [11]. In the presence of a Coulomb field $V$ and a magnetic field $\mathbf{B}_{A}$ generated by a vector potential $\mathbf{A}$, this operator is given by (in relativistic units, $\hbar=c=1$ )

$$
H=D_{A}+V, \quad D_{A}=\boldsymbol{\alpha}(\mathbf{p}-e \mathbf{A})+\beta m, \quad V=-\frac{\gamma}{x} .
$$

In this expression $\boldsymbol{\alpha}$ and $\beta$ are the Dirac matrices, $\mathbf{p}=-i \boldsymbol{\nabla}$ is the momentum operator, $x=|\mathbf{x}|$ and $\gamma=Z e^{2}$ is the electric potential strength ( $Z$ is the charge of the point-like nucleus which is fixed at the origin and $e^{2} \approx 1 / 137.036$ is the fine structure constant).

One way to avoid dealing with the negative continuum, which, in contrast to the nonrelativistic case, causes the Dirac operator to be unbounded from below, is the introduction of semibounded pseudorelativistic operators such as the Herbst operator [5]. This operator was originally put forth by Chandrasekhar (see e.g. $[12])$ and acts in the Hilbert space $L_{2}\left(\mathbb{R}^{3}\right) \otimes \mathbb{C}^{2}$,

$$
\begin{gathered}
h^{H}=E_{A}+V, \\
E_{A}=\left|D_{A}\right|=\sqrt{(\boldsymbol{\sigma}(\mathbf{p}-e \mathbf{A}))^{2}+m^{2}},
\end{gathered}
$$

where $\boldsymbol{\sigma}$ is the vector of Pauli spin matrices. For $\mathbf{A} \in L_{2, l o c}\left(\mathbb{R}^{3}\right)$ the form domain of $E_{A}$ is the Sobolev space $H_{1 / 2}\left(\mathbb{R}^{3}\right) \otimes \mathbb{C}^{2}$. It was shown in [4] that $h^{H}$ is bounded 
from below if $\gamma<\frac{2}{\pi}(Z \leq 87)$ and if $\mathbf{B}_{A}$ is locally bounded. Under these conditions $h^{H}$ can be extended to a self-adjoint operator.

In the following we assume that the field $\mathbf{B}_{A}$ is generated by a two-dimensional vector potential $\mathbf{A}=\left(A_{1}, A_{2}, 0\right)$ taken to be independent of $x_{3}$ and obeying $\boldsymbol{\nabla} \cdot \mathbf{A}=0$. Then the kinetic energy can be decomposed in the following way,

$$
E_{A}^{2}=E_{x y}^{2}+\left(p_{3}^{2}+m^{2}\right), \quad E_{x y}^{2}=\sum_{k=1}^{2}\left(p_{k}-e A_{k}\right)^{2}-e \sigma_{3} B_{A} .
$$

For a given trial function $\psi_{t} \in H_{1 / 2}\left(\mathbb{R}^{3}\right) \otimes \mathbb{C}^{2}$, normalized to unity, we have by the Schwarz inequality the estimate for the ground-state energy $E_{g}$ of the Herbst operator,

$$
E_{g} \leq\left(\psi_{t}, h^{H} \psi_{t}\right) \leq \sqrt{\left(\psi_{t}, E_{A}^{2} \psi_{t}\right)}+\left(\psi_{t}, V \psi_{t}\right)=: E^{H}\left[\psi_{t}\right]
$$

We wish to discuss the Herbst operator for two classes of locally bounded magnetic fields $\mathbf{B}_{A}$, the first being of asymptotic growth,

$$
\mathbf{B}_{A_{1}}(\mathbf{x})=B \frac{1+\tau}{2}\left(0,0,\left|x_{1}\right|^{\tau}+\left|x_{2}\right|^{\tau}\right),
$$

characterized by the parameters $B$ and $\tau \geq 0$, where $\tau=0$ corresponds to a constant magnetic field of strength $B$ in the direction of the $x_{3}$-axis. For this class of fields it was proven (for $0.1 \lesssim \gamma<\frac{2}{\pi}$ and large $B$ ) that a bound ground state exists when $\tau$ is subcritical, $\tau<\tau_{c}$, where $\tau_{c}$ depends on $Z$ as well as on $B$ [4].

The suppression of the magnetic force around the origin (being the stronger the larger $\tau$ ) can also be described by the (simpler) second class of magnetic fields. This class was introduced by Egger and coworkers in the context of the two-dimensional electronic motion [1],

$$
\mathbf{B}_{A_{2}}(\mathbf{x})=B \theta\left(\varrho-r_{0}\right) \mathbf{e}_{3}, \quad \varrho=\sqrt{x_{1}^{2}+x_{2}^{2}},
$$

where $\theta$ is Heaviside's step function. It is parametrized by the field strength $B$ and the radius $r_{0}$ of the field-free region. The constant magnetic field is included in the class $(2.6)$ for $r_{0}=0$. The energy functionals pertaining to the fields (2.5) and (2.6) will be denoted by $E^{H 1}\left[\psi_{t}\right]$ and $E^{H 2}\left[\psi_{t}\right]$, respectively.

As trial function we take, following Rau et al [7],

$$
\begin{gathered}
\psi_{t}(\mathbf{x})=N_{t} e^{-\nu^{2} \varrho^{2} / 2} e^{-Z^{\prime} x}\left(\begin{array}{l}
1 \\
0
\end{array}\right), \\
N_{t}=\left[\frac{\nu^{4}}{Z^{\prime} \pi} \frac{1}{\psi\left(2,2, Z^{\prime 2} / \nu^{2}\right)}\right]^{\frac{1}{2}},
\end{gathered}
$$

where the spin direction is taken parallel to $\mathbf{B}_{A}$. In the normalization constant $N_{t}, \psi(n, k, \xi)$ is the irregular confluent hypergeometric function which is readily expressed in terms of the integral representation [13, p.1058],

$$
\psi(n, k, \xi)=\frac{1}{\Gamma(n)} \int_{0}^{\infty} d t e^{-\xi t} \frac{t^{n-1}}{(1+t)^{n+1-k}} .
$$


In (2.7), $\nu=\sqrt{2 s}(e B)^{d / 2}$ measures the inverse extension of the electron orbit perpendicular to $\mathbf{B}_{A}$, where $d>0$ is a field-specific constant. Besides the effective charge $Z^{\prime}$ we have introduced $s$ as a second variational parameter $\left(s=\frac{1}{4}\right.$ for constant magnetic fields). Thus the trial function mimicks an eigenstate for the lowest Landau level (in the case of vanishing scalar potential and constant magnetic field) as well as the hydrogenic ground state (in the case of zero magnetic field). $E^{H}\left[\psi_{t}\right]$,

The ground-state energy is estimated by the infimum of the energy functional

$$
E_{g}^{H}:=\inf _{Z^{\prime}>0, s>0} E^{H}\left[\psi_{t}\right] .
$$

It is easy to show that for vanishing magnetic field $E_{g}^{H}$ agrees with the exact Dirac ground-state energy. Since for $B_{A}=0$ the trial function reduces to $\psi_{t}(\mathbf{x})=$ $\left(Z^{\prime} 3 / 2 / \pi^{\frac{1}{2}}\right) e^{-Z^{\prime} x}\left(\begin{array}{l}1 \\ 0\end{array}\right)$, we get

$$
\begin{gathered}
E^{H}\left[\psi_{t}\right]=\sqrt{\left(\psi_{t},\left(p^{2}+m^{2}\right) \psi_{t}\right)}-\gamma\left(\psi_{t}, \frac{1}{x} \psi_{t}\right) \\
=\sqrt{Z^{\prime 2}+m^{2}}-\gamma Z^{\prime} .
\end{gathered}
$$

From $\partial E^{H} / \partial Z^{\prime}=0$ we obtain $Z^{\prime}=m \gamma / \sqrt{1-\gamma^{2}}$ and thus

$$
E_{g}^{H}\left(B_{A}=0\right)=\inf _{Z^{\prime}>0} E^{H}\left[\psi_{t}\right]\left(B_{A}=0\right)=m \sqrt{1-\gamma^{2}},
$$

which is equal to the exact Dirac energy.

Moreover, the variationally determined ground-state energy for a constant magnetic field compares well with the available results for $Z>10$ from elaborate relativistic calculations $[14,15]$. Thereby it is advantageous to subtract the rest energy of the electron, i.e. to consider $E_{g-}^{H}=E_{g}^{H}-m$. Table 1 gives the comparison for $Z=20$. For $\lambda=B / Z^{2} \geq 10$, the reference values are obtained from the Schrödinger scaling (see below) of the exact results.

\begin{tabular}{c|l|l|l|}
$\lambda$ & $E_{g-}^{H}$ & $E_{g-}^{e x}$ & $E_{g-}^{e x s}$ \\
\hline 0.025 & -205.90 & -205.98 & \\
0.25 & -244.61 & -244.95 & \\
2 & -407.65 & -409.55 & \\
10 & -690.89 & & -699.16 \\
20 & -871.44 & & -886.16 \\
200 & -1841.75 & & -1890.85 \\
500 & -2436.99 & & -2502.81 \\
2000 & -3635.26 & & -3721.84
\end{tabular}

Table 1. Ground-state energy (rest energy subtracted, in atomic units) for $Z=20$ as a function of $\lambda=B / Z^{2}$ for a constant magnetic field of strength $B$. The second column gives the results from the present calculation, the third column comprises the exact results $[14,15] . E_{g-}^{e x s}$ is obtained from the Schrödinger scaling 
of the exact result for $Z=5$ at $\lambda=10$ [16] and for $Z=1$ when $\lambda \geq 20$ [14]. $B$ is given in units of $B_{0}=2.35 \times 10^{9} \mathrm{G}$.

There is one additional exact calculation for uranium $\left(E_{g-}^{e x}=-4861.61 \mathrm{a} . \mathrm{u}\right.$. [15] but for a very small field, $B=1$ (relating to $\lambda=1.182 \times 10^{-4}$ ), where the numerical inaccuracy of our result $\left(E_{g-}^{H}=-4860.05\right.$ a.u. $)$ is quite large $(\sim 2$ a.u. $)$.

\subsection{Magnetic fields with asymptotic growth.}

Magnetic fields of the class (2.5) are generated by the vector potentials

$$
\mathbf{A}_{1}(\mathbf{x})=\frac{B}{2}\left(-x_{2}\left|x_{2}\right|^{\tau}, x_{1}\left|x_{1}\right|^{\tau}, 0\right), \quad \tau \geq 0
$$

In the trial function $(2.7)$ we take $d=\frac{2}{2+\tau}$. This choice preserves the scaling property of the Herbst operator [4]. We decompose

$$
\left(\psi_{t}, E_{A}^{2} \psi_{t}\right)=M_{a n}+M_{n u m}^{(1)},
$$

where $M_{a n}$ is the analytic part,

$$
M_{a n}=2 \nu^{2}-Z^{\prime 2}+m^{2}-\nu^{4}\left(\psi_{t}, \varrho^{2} \psi_{t}\right)-2 \nu^{2} Z^{\prime}\left(\psi_{t}, \frac{\varrho^{2}}{x} \psi_{t}\right)+2 Z^{\prime}\left(\psi_{t}, \frac{1}{x} \psi_{t}\right),
$$

while $M_{\text {num }}^{(1)}$ is the $B$-dependent part to be evaluated numerically,

$$
\begin{aligned}
M_{\text {num }}^{(1)} & =\left(\frac{e B}{2}\right)^{2}\left(\psi_{t},\left(\left|x_{1}\right|^{2+2 \tau}+\left|x_{2}\right|^{2+2 \tau}\right) \psi_{t}\right) \\
& -e B \frac{1+\tau}{2}\left(\psi_{t},\left(\left|x_{1}\right|^{\tau}+\left|x_{2}\right|^{\tau}\right) \psi_{t}\right) .
\end{aligned}
$$

Using the cylindrical symmetry of $\psi_{t}$ and spherical coordinates $(x, \vartheta, \varphi)$ we have

$$
\begin{gathered}
\left(\psi_{t},\left|x_{1}\right|^{\tau} \psi_{t}\right)=\left(\psi_{t},\left|x_{2}\right|^{\tau} \psi_{t}\right)=2 N_{t}^{2} \int_{0}^{\infty} d x x^{2} e^{-2 Z^{\prime} x} \\
\cdot \int_{0}^{1} d(\cos \vartheta) e^{-\nu^{2} x^{2} \sin ^{2} \vartheta} x^{\tau}(\sin \vartheta)^{\tau} \int_{-\pi}^{\pi} d \varphi|\cos \varphi|^{\tau} .
\end{gathered}
$$

With the substitution $y=\sin ^{2} \vartheta$ we obtain [13, p.318,369]

$$
\left(\psi_{t},\left(\left|x_{1}\right|^{\tau}+\left|x_{2}\right|^{\tau}\right) \psi_{t}\right)=8 \pi N_{t}^{2} \frac{1}{1+\tau} \int_{0}^{\infty} d x x^{2+\tau} e^{-2 Z^{\prime} x} e^{-\nu^{2} x^{2}}{ }_{1} F_{1}\left(\frac{1}{2}, \frac{3+\tau}{2}, \nu^{2} x^{2}\right)
$$

where ${ }_{1} F_{1}(a, b, z)$ is the (regular) confluent hypergeometric function.

The matrix elements in (2.14) are evaluated in a similar way. With the help of $x e^{-2 Z^{\prime} x}=-\frac{1}{2} \frac{d}{d Z^{\prime}}\left(e^{-2 Z^{\prime} x}\right)$ we find [13, p.867] (see also [7])

$$
\left(\psi_{t}, \varrho^{2} \psi_{t}\right)=-\frac{Z^{\prime}}{\nu^{6}} \pi N_{t}^{2} \psi^{\prime}\left(2,1, \frac{Z^{\prime 2}}{\nu^{2}}\right), \quad\left(\psi_{t}, \frac{\varrho^{2}}{x} \psi_{t}\right)=\frac{1}{\nu^{4}} \pi N_{t}^{2} \psi\left(2,1, \frac{Z^{\prime 2}}{\nu^{2}}\right)
$$


and

$$
\left(\psi_{t}, \frac{1}{x} \psi_{t}\right)=\frac{\pi}{\nu^{2}} N_{t}^{2} \psi\left(1,1, \frac{Z^{\prime 2}}{\nu^{2}}\right)
$$

which also determines the potential energy, $\left(\psi_{t},-\frac{\gamma}{x} \psi_{t}\right)$. The derivative $\psi^{\prime}(n, k, \xi)$ with respect to $\xi$ is readily obtained from the integral representation (2.8).

For the discussion of the $Z$ - and $B$-dependence of the variationally determined ground-state energy it is convenient to introduce the parameter $\lambda=B / Z^{2}$. In the case of a constant magnetic field $\lambda$ provides the ratio between the magnetic and electric field strengths acting on the electron [7]. For Schrödinger operators there is an exact scaling which allows to express the ground-state energy divided by $Z^{2}$ just in terms of $\lambda[17,14]$. Also for relativistic systems this scaling is satisfied quite well up to $\lambda \sim 10^{3}[9]$. Therefore the basic physics can be displayed with a single choice of $Z$.

Fig.2a shows the ground-state energy $E_{g-}^{H 1}$ for $Z=80$ as a function of the asymptotic growth $\tau$. Clearly, for a constant magnetic field $(\tau=0) \quad E_{g-}^{H 1}$ is decreasing with $B$. At large $\tau$, on the other hand, the variational ground-state energy tends to the exact Dirac energy in the absence of a magnetic field (i.e. (2.11), with rest energy subtracted). This can readily be explained by the fact that large $\tau$ correspond to a near-zero magnetic field in an extended region around the nucleus. Note that for small magnetic field strengths $E_{g-}^{H 1}$ approaches this asymptotic value from below, while at the higher $B$ a maximum evolves, such that eventually the asymptotic energy is approached from above.

When one introduces (for $B \neq 0$ ) the scaled parameters $\tilde{Z}=Z^{\prime} / \nu$ and $\tilde{m}_{s}=m / \nu$ one can show that the energy functional $E^{H 1}\left[\psi_{t}\right]$ scales with the field strength $B$ according to $\nu \sim B^{d / 2}$. In particular, with $d=\frac{2}{2+\tau}$, the variationally determined ground-state energy can be written in the following way,

$$
E_{g-}^{H 1}=E_{g}^{H 1}-m=\nu\left(\tilde{E}_{g}^{H 1}-\tilde{m}_{s}\right) \sim B^{\frac{1}{2+\tau}} \tilde{E}_{g-}^{H 1},
$$

where $\tilde{E}_{g-}^{H 1}$ depends on $B$ only through the scaled mass $\tilde{m}_{s}$. In turn, $\tilde{m}_{s}$ influences the optimized parameters $\tilde{Z}$ and $s$ (which, since $\tilde{m}_{s} \rightarrow 0$ as $B \rightarrow \infty$, tend to constant values as $B \rightarrow \infty)$. Based on the existence of a bound ground state (for small $\tau$ ) it was proven in [4] that also the exact ground-state energy of $h^{H}$ decreases with $B$ according to $B^{\frac{1}{2+\tau}}$ when $B \rightarrow \infty$.

Fig.2b depicts the scaled ground-state energies $E_{g-}^{H 1} / \lambda^{\frac{1}{2+\tau}}$ of Fig.2a, including the limiting case $\tilde{m}_{s}=0$ (which corresponds to $B=\infty$ ). It can be strictly proven [4] and is verified in the figure that the scaled energy increases with $B$ (i.e. decreases with $\left.m / B^{1 /(2+\tau)}\right)$. All curves show a maximum near $\tau=1.5$ which becomes positive when $\lambda>10^{6}$ (corresponding to $B>10^{6} Z^{2} B_{0}$ with $B_{0}=2.35 \times 10^{9} \mathrm{G}$ the unit field). For $\lambda$ fixed and $\tau \rightarrow \infty$ the scaled energy approaches the Dirac energy too (since $\lim _{\tau \rightarrow \infty} E_{g-}^{H 1} / \lambda^{\frac{1}{2+\tau}}=\lim _{\tau \rightarrow \infty} E_{g-}^{H 1}$ ). 


\subsection{Egger-type magnetic fields.}

In this section we consider a magnetic field which is constant outside a cylinder of radius $r_{0}$ centered around the $x_{3}$-axis, and zero inside. This field, given by (2.6), is generated by the vector potential [3]

$$
\mathbf{A}_{2}(\mathbf{x})=\frac{B}{2}\left(1-\frac{r_{0}^{2}}{\varrho^{2}}\right) \theta\left(\varrho-r_{0}\right)\left(-x_{2}, x_{1}, 0\right) .
$$

In order to preserve the scaling property we have to set $d=1$ (corresponding to $\tau=0$ in the field $\left.\mathbf{A}_{1}\right)$, such that in the trial function $(2.7), \nu=\sqrt{2 s e B}$.

The field-dependent part of the energy functional $E^{H 2}\left[\psi_{t}\right]$ is most readily evaluated when cylindrical coordinates $\left(\varrho, \varphi, x_{3}\right)$ are used. With the help of the integral

$$
\int_{-\infty}^{\infty} d x_{3} e^{-2 Z^{\prime} \sqrt{\varrho^{2}+x_{3}^{2}}}=2 \varrho K_{1}\left(2 \varrho Z^{\prime}\right),
$$

where $K_{1}$ is a modified Bessel function, we get

$$
M_{n u m}^{(2)}=4 \pi N_{t}^{2} \int_{r_{0}}^{\infty} d \varrho e^{-\nu^{2} \varrho^{2}} K_{1}\left(2 \varrho Z^{\prime}\right)\left[\left(\frac{e B}{2}\right)^{2}\left(\varrho^{2}-r_{0}^{2}\right)^{2}-e B \varrho^{2}\right] .
$$

Thus, with (2.4) and (2.13),

$$
E^{H 2}\left[\psi_{t}\right]=\sqrt{M_{a n}+M_{n u m}^{(2)}}+\left(\psi_{t}, V \psi_{t}\right) .
$$

In Fig.3a the ground-state energy $E_{g-}^{H 2}$, resulting from the infimum of (2.24) with respect to $Z^{\prime}$ and $s$, is plotted for fixed $\lambda$ as a function of $\tilde{d}=r_{0} \sqrt{\lambda}$. In this representation the curves are very similar to those shown in Fig.2a. In particular, the maximum (which appears for sufficiently high field strengths) is also at a fixed position, $\tilde{d} \approx 3$. Again, the field-free Dirac energy is approached when $\tilde{d} \rightarrow \infty$.

When the scaling with $\nu$ is introduced, such that $\varrho$ is replaced by $\tilde{\varrho}=\varrho \nu$, the hole diameter $r_{0}$ changes into $r_{0} \nu$ which increases according to $B^{\frac{1}{2}}$. Thus $\tilde{d}$ from Fig.3a can be interpreted as the scaled hole diameter in units of the $K$-shell radius, $1 / Z$. Fig. 3b displays the scaled energy $E_{g-}^{H 2} / \lambda^{\frac{1}{2}}$ for a wide range of $\lambda$ as a function of $\tilde{d}$. Again, this $r_{0}$-dependence resembles the $\tau$-dependence of $E_{g-}^{H 1} / \lambda^{\frac{1}{1+\tau}}$ from Fig.2b, with two minor exceptions: The maximum becomes positive for $\lambda>48.9$ which is much lower than the corresponding value in Fig. $2 \mathrm{~b} \quad\left(\lambda>10^{6}\right)$. Also, the behaviour for $B \rightarrow \infty$ is different. While the curves for $\lambda=10^{8}$ and $\lambda=\infty$ nearly coincide in Fig.3b, they differ considerably in Fig.2b. This is related to the additional $B$-dependence of the abscissa in Fig.3b. The range $0 \leq \tilde{d} \leq 30$ corresponds to a nearly homogeneous field $\left(r_{0} \approx 0\right)$ for $\lambda \gtrsim 10^{6}$ such that the convergence with $\lambda \rightarrow \infty$ mimicks the fast converence of the scaled ground-state energy with $\tilde{m}_{s} \rightarrow 0$ for $r_{0}=0$. The convergence proof from [4], based on the continuity of $E_{A}$ as a function of $m$, holds for any $\tau>0$. However, when the depleted region is large (Fig.2b), the convergence with $\tilde{m}_{s}$ becomes slow. In this 
context we also note that the scaled energy $E_{g-}^{H 2} / \lambda^{1 / 2}$ tends to a constant (for $\left.r_{0} \rightarrow \infty\right)$ which, in contrast to $E_{g-}^{H 1} / \lambda^{1 /(2+\tau)}$ (for $\left.\tau \rightarrow \infty\right)$, decreases with $\lambda$.

\section{Comparison with previous Results for $B \rightarrow \infty$}

When the strength of a homogeneous magnetic field is very large $(\lambda \gg 1)$, the confinement of the electron in the direction perpendicular to $\mathbf{B}_{A}$ is given by the cyclotron radius $a_{0}=\sqrt{\frac{2}{e B}}$ (see e.g. [7]). Assuming that this is also true for small $\tau$ we have in our earlier work [4] taken a separable trial function where in the hydrogenic part $\varphi_{z}$ the coordinate $\varrho$ is replaced by $a_{0}=1 / \nu=\frac{1}{\sqrt{2 s(e B)^{2 /(2+\tau)}}}$,

$$
\begin{gathered}
\psi_{t}^{(1)}(\mathbf{x})=\frac{\nu}{\sqrt{\pi}} e^{-\nu^{2} \varrho^{2}} \varphi_{z}\left(x_{3}\right)\left(\begin{array}{l}
1 \\
0
\end{array}\right), \\
\varphi_{z}\left(x_{3}\right)=\left(2 a_{0} K_{1}\left(2 a_{0} Z^{\prime}\right)\right)^{-\frac{1}{2}} e^{-Z^{\prime} \sqrt{a_{0}^{2}+x_{3}^{2}}} .
\end{gathered}
$$

Consequently, a separable kinetic energy functional was taken, based on the inequality

$$
\left(\psi, E_{A} \psi\right) \leq \sqrt{\left(\psi, E_{x y}^{2} \psi\right)}+\left(\psi, \sqrt{p_{3}^{2}+m^{2}} \psi\right)=: E_{s e p}[\psi]
$$

for any normalized $\psi \in H_{1 / 2}\left(\mathbb{R}^{3}\right) \otimes \mathbb{C}^{2}$. The estimate for the ground-state energy was obtained from minimizing the energy functional

$$
E_{\text {sep }}^{H 1}\left[\psi_{t}^{(1)}\right]:=E_{\text {sep }}\left[\psi_{t}^{(1)}\right]+\left(\psi_{t}^{(1)}, V \psi_{t}^{(1)}\right)
$$

relating to the field $\mathbf{B}_{A_{1}}$, with respect to $Z^{\prime}$ and $s$. For large $\tau$ this functional is expected to be inferior to $E^{H 1}\left[\psi_{t}\right]$ from Section 2, because $\mathbf{B}_{A_{1}}$ contains an extended depleted region where $\psi_{t}^{(1)}$ fails. This is confirmed for the limiting case $\tilde{m}_{s}=0$ (i.e. $B=\infty$ ) in Fig. 4 where the scaled energies from the two functionals are compared for $\tau \lesssim 10$. The two curves cross near $\tau=0.25$, and $E^{H 1}\left[\psi_{t}\right]$ provides indeed the smaller energy estimate for all $\tau$ that exceed this value.

Since, however, two different functionals are used for the kinetic energy, both being upper bounds for $\left(\psi, E_{A} \psi\right)$, one may ask how the results will change when these functionals are interchanged while keeping the trial function fixed. Correspondingly, we define the two additional energy functionals $E_{\text {sep }}^{H 1}\left[\psi_{t}\right]$ with $\psi_{t}$ from (2.7) as well as $E^{H 1}\left[\psi_{t}^{(1)}\right]$ with the kinetic energy estimate from (2.4). The minimization of $E_{\text {sep }}^{H 1}\left[\psi_{t}\right]$ proves to be inferior at all $\tau$ investigated (see Fig.4), whereas the use of a separable trial function together with the non-separable energy functional indeed provides the lowest energy estimate when $\tau>0.2$. Thus a separable trial function (together with an appropriate energy functional) is the best choice at $B \rightarrow \infty$ for all values of $\tau$. We note that this remains true for finite (but high) field strengths, provided $\tau$ is not too large (see Fig.2b).

With the help of the functional $E^{H 1}\left[\psi_{t}^{(1)}\right]$ the critical field growth $\tau_{c}$ below which the energy estimate is negative (hence guaranteeing the existence of a bound ground state) can be improved from $\tau_{c}=0.602$ [4] to $\tau_{c}=0.943$ (for $Z=80$ ). 


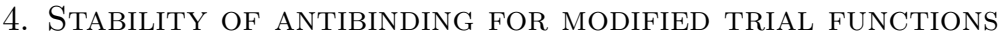

The infimum $E_{g}^{H}$ of the energy functional provides only an upper bound to the exact ground-state energy of the Herbst operator. In order to assure that the ground-state energy is indeed positive in a certain parameter range we apply trial functions of different type and study their influence on the variational energy in the case of the Egger-type field (2.6). Guided by the fact that for $B=0$ the variationally determined ground-state energy of the related Brown-Ravenhall operator is lowered when the trial function accounts for the relativistic contraction [9], we consider the energy functional $E^{H}\left[\psi_{t}^{(2)}\right]$ from (2.4) with

$$
\psi_{t}^{(2)}(\mathbf{x})=N_{2} e^{-\nu^{2} \varrho^{2} / 2} x^{\tilde{\gamma}} e^{-Z^{\prime} x}\left(\begin{array}{l}
1 \\
0
\end{array}\right), \quad \tilde{\gamma}=\sqrt{1-\left(Z^{\prime} e^{2}\right)^{2}}-1
$$

where $\nu=\sqrt{2 s e B}$ as before. The normalization constant can be calculated along the lines of (2.16) and (2.17),

$$
N_{2}=\left(4 \pi \int_{0}^{\infty} d x x^{2+2 \tilde{\gamma}} e^{-2 Z^{\prime} x} e^{-\nu^{2} x^{2}}{ }_{1} F_{1}\left(\frac{1}{2}, \frac{3}{2}, \nu^{2} x^{2}\right)\right)^{-\frac{1}{2}} .
$$

As an alternative to $\tilde{\gamma}$ determined by $Z^{\prime}$, we have also considered $\tilde{\gamma}$ of the form $\tilde{\gamma}(\zeta)=\sqrt{1-\left(\zeta e^{2}\right)^{2}}-1$, related to an independent variational parameter $\zeta$ (besides $Z^{\prime}$ and $\left.s\right)$.

Our results from the variation with respect to $Z^{\prime}$ and $s$ are displayed in Fig.5a. We have plotted the scaled energies $E_{g-}^{H 2} / \sqrt{\lambda}$, obtained from the functions $\psi_{t}$ and $\psi_{t}^{(2)}$, respectively, versus the true hole diameter $r_{0}=\tilde{d} / \sqrt{\lambda}$. In this representation it becomes clear that the maximum of the energy shifts to smaller $r_{0}$ when the field strength increases. For the test cases $\lambda=10^{2}$ and $10^{4}$, relating to a positive maximum, the energy derived from the function (4.1) is always higher than the energy obtained in section 2.2. This fact remains unchanged when $\zeta$ is introduced as a third variational parameter: Only for weak fields ( $\operatorname{such}$ as $\lambda=2$ and $\tilde{d} \gtrsim 3$ ) is the energy slightly lower when $\zeta>0$ (the deviation from the $\zeta=0$ results being below 1 percent).

When the field is switched off completely and correspondingly the factor $\exp \left(-\nu^{2} \varrho^{2} / 2\right)$ omitted from the trial function (4.1) (such that the normalization constant reduces to $\left.N_{2}=\left(2 Z^{\prime}\right)^{\frac{3}{2}+\tilde{\gamma}} / \sqrt{4 \pi \Gamma(3+2 \tilde{\gamma})}\right)$, the energy functional is given by

$$
E_{-}^{H}\left[\psi_{t}^{(2)}\right]=\left(m^{2}+\frac{Z^{\prime 2}}{1+2 \tilde{\gamma}}\right)^{\frac{1}{2}}-m-\gamma \frac{Z^{\prime}}{1+\tilde{\gamma}} .
$$

It turns out that its minimum is again higher than if $\tilde{\gamma}$ is set equal to zero (for $Z=80$, one gets $E_{g-}^{H}=-3382.98$ a.u. as compared to -3532.19 a.u.). When $\zeta$ is treated as independent variational parameter, the minimum is obtained for $\tilde{\gamma}=0$.

We have also considered the case where a positive power of the radial coordinate is introduced into the trial function. In fact, when the scalar potential is 
absent $(Z=0)$ and the magnetic field homogeneous, the ground state of the electron is infinitely degenerate with eigenstates relating to different powers of $\varrho$ [11]. When the magnetic field is kept homogeneous but the scalar potential is turned on the degeneracy is lifted, the energy increasing with increasing power of $\varrho[7,6]$. For the investigation in the case of inhomogeneous fields we use the following trial function,

$$
\begin{gathered}
\psi_{t}^{(3)}(\mathbf{x})=N_{3} e^{-\nu^{2} \varrho^{2} / 2}\left(\varrho e^{i \varphi}\right)^{l} e^{-Z^{\prime} x}\left(\begin{array}{l}
1 \\
0
\end{array}\right), \\
N_{3}=\left(-\frac{\pi Z^{\prime} \Gamma^{2}(l+1)}{\nu^{2 l+4}} \psi^{\prime}\left(l+1,1, \frac{Z^{\prime 2}}{\nu^{2}}\right)\right)^{-\frac{1}{2}}
\end{gathered}
$$

where $N_{3}$ is the normalization constant, $\nu=\sqrt{2 s e B}$ and $\psi^{\prime}$ the derivative of the irregular confluent hypergeometric function.

The $B$-independent part of the energy functional (2.4) can for $\psi_{t}^{(3)}$ still be evaluated analytically [7], whereas the $B$-dependent part is given by $(2.23)$, modified by the additional factor $\varrho^{2 l}$ in the integrand, plus a nonvanishing contribution from the cross term,

$$
\begin{gathered}
\left(\psi_{t}^{(3)},\left(-2 e A_{1} p_{1}-2 e A_{2} p_{2}\right) \psi_{t}^{(3)}\right) \\
=-4 \pi l e B N_{3}^{2} \int_{r_{0}}^{\infty} d \varrho \varrho^{2 l} e^{-\nu^{2} \varrho^{2}}\left(\varrho^{2}-r_{0}^{2}\right) K_{1}\left(2 \varrho Z^{\prime}\right) .
\end{gathered}
$$

If $l>0$ is fixed, the minimum of this energy functional, $E^{H 2}\left[\psi_{t}^{(3)}\right]$, is indeed higher than in the case of the variational function $\psi_{t}$ from (2.7) and increases with $l$. The effect is particularly large when $l$ is restricted to integers like in the degenerate $Z=r_{0}=0$ eigenstates. For $l=0.1$ and 1 this is shown in Fig.5b where the scaled energy near its maximum (at $\tilde{d}=3$ ) is plotted as a function of the field strength. Included are the $l=0$ results for $Z=20$ to display the $Z$-dependent monotonous increase of the scaled energy with $\lambda$ at fixed $\tilde{d}$ up to saturation for $\lambda \gtrsim 10^{8}$.

\section{Conclusion}

We have studied the ground-state energy of an atomic electron in an inhomogeneous magnetic field which is described by two parameters, the size of a field-free area around the nucleus and the field strength. Irrespective of the particular choice of the magnetic field the variational estimate of the ground-state energy of the Herbst operator, used to model the relativistic electron, becomes positive for a certain limited size of this field-free region if the field strength is larger than some critical value. The lowest energy estimate is obtained for a trial function which combines an eigenfunction of the lowest Landau level with a nonrelativistic hydrogenic function. The only exceptions are ultrastrong fields, including the limit $B \rightarrow \infty$, where a trial function, which is separable in the coordinates parallel and perpendicular to the magnetic field, is more appropriate. 
Our conjecture that the antibinding of the electron in a particular parameter range is real and not an artefact due to an inappropriate choice of the trial function is supported by two facts. First, a positive maximum of the variational groundstate energy is obtained for all trial functions investigated. Second, this maximum increases with a positive power of the field strength. Thus we have established the possibility of static ionization of a heavy ion by means of an appropriately chosen strong inhomogeneous magnetic field.

\section{ACKNOWLEDGMENT}

I would like to thank H.Siedentop for stimulating this project, and R.Egger for detailed discussions of his work. Support from the DFG (SFB/TR12) is gratefully acknowledged.

\section{REFERENCES}

[1] A.De Martino, L.Dell'Anna. and R.Egger, Magnetic confinement of massless Dirac fermions in graphene, Phys. Rev. Lett. 98, 066802 (2007).

[2] A.De Martino and R.Egger, On the spectrum of a magnetic quantum dot in graphene, Semicond. Sci. Technol. 25, 034006 (2010).

[3] R.Egger, A.De Martino, H.Siedentop and E.Stockmeyer, Multiparticle equations for interacting Dirac fermions in graphene nanostructures, J. Phys. A 43, 215202 (2010).

[4] D.H.Jakubassa-Amundsen, Scaling of the ground-state energy of relativistic ions in high locally bounded magnetic fields, J. Math. Phys. 51, 082303 (2010).

[5] I.W.Herbst, Spectral theory of the operator $\left(p^{2}+m^{2}\right)^{\frac{1}{2}}-Z e^{2} / r$, Commun. Math. Phys. 53, 285-294 (1977).

[6] D.Lai, Rev. Mod. Phys. 73, 629-661 (2001).

[7] A.R.P.Rau, R.O.Mueller and L.Spruch, Simple model and wave function for atoms in intense magnetic fields, Phys. Rev. A 11, 1865-1879 (1975).

[8] A.K.Rajagopal, G.Chanmugam, R.F.O'Connell and G.L.Surmelion, Phys. Rev. D 6, 3700 (1972).

[9] D.H.Jakubassa-Amundsen, Variational ground state for relativistic ions in strong magnetic fields, Phys. Rev. A 78, 062103, 1-9 (2008).

[10] D.H.Jakubassa-Amundsen, The ground state of relativistic ions in the limit of high magnetic fields, Ann. Henri Poincaré 10, 1207-1222 (2009).

[11] B.Thaller, The Dirac Equation (Springer, Berlin, 1992).

[12] S.Chandrasekhar, On stars, their evolution and their stability, Rev. Mod. Phys. 56, 137-147 (1984).

[13] I.S.Gradshteyn and I.M.Ryzhik, Table of Integrals, Series and Products (Academic, New York, 1965).

[14] S.P.Goldman and Z.Chen, Generalized relativistic variational calculations for hydrogenic atoms in arbitrary magnetic fields, Phys. Rev. Lett. 67, 1403 (1991).

[15] Z.Chen and S.P.Goldman, Phys. Rev. A 48, 1107 (1993).

[16] Z.Chen and S.P.Goldman, Phys. Rev. A 44, 4459 (1991).

[17] G.L.Surmelian and R.F.O'Connell, Astrophys. J. 190, 741 (1974). 


\section{Figure Captions}

Fig.1

(a) Cylindrically symmetric Egger-type magnetic field, $\varrho=\sqrt{x_{1}^{2}+x_{2}^{2}}$.

(b) Magnetic field with asymptotic growth in the special case of cylindrical symmetry $(\tau=2)$.

Fig.2

Ground-state energy (without rest energy) for $Z=80$ and the magnetic field (2.5) as a function of $\tau$. The parameter $\lambda$ labelling the curves relates to the field strength according to $B=\lambda Z^{2}$ (in units of $B_{0}=2.35 \times 10^{9} \mathrm{G}$ ).

(a) Unscaled energy $E_{g-}^{H 1}$ (in atomic units) for $\lambda=100(-), 10^{6}\left(----_{-}\right)$ and $10^{8}(-\cdot-\cdot-)$. The asymptotic value is the Dirac energy for $B=0,-3532.19$ a.u.

(b) Scaled energy $E_{g-}^{H 1} / \lambda^{1 /(2+\tau)}$ for $\lambda=100(--), 10^{6}(----), 10^{10}(-\cdot$ $-\cdot-)$ and $\infty$ (functional $E^{H 1}\left[\psi_{t}^{(1)}\right](*$, see Section 3$)$.

Fig.3

Ground-state energy (without rest energy) for $Z=80$ and the Egger field (2.6) as a function of the scaled hole diameter $\tilde{d}=r_{0} \sqrt{\lambda}$ (in units of the $K$-shell radius, $1 / Z$ a.u.).

(a) Unscaled energy $E_{g-}^{H 2}$ (in atomic units) for $\lambda=2(-----), 30(-)$ and $100(-\cdot-\cdot-)$. The horizontal line marks the Dirac energy for $B=0,-3532.19$ a.u.

(b) Scaled energy $E_{g-}^{H 2} / \lambda^{1 / 2}$ for $\lambda=2\left(----^{-}\right), 30$ (- -100 (- . $-\cdot-), 10^{4}(\cdots \cdots)$ and $10^{8}$ (— uppermost curve). Included are results for $\lambda=\infty(\times)$.

Fig. 4

Scaled ground-state energy $E_{g-}^{H 1} / \lambda^{1 /(2+\tau)}$ (without rest energy) for the magnetic field (2.5) of infinite strength $(B=\infty)$ and $Z=80$ as a function of $\tau$. Results are shown for different trial functions and kinetic energy operators: $\psi_{t}$ with $(2.4)$, see also Fig.2b (- $) ; \psi_{t}^{(1)}$ with $(3.2)(-----) ; \psi_{t}$ with $(3.2)(\times)$ and $\psi_{t}^{(1)}$ with $(2.4)(*)$.

Fig.5

Scaled ground-state energy $E_{g-}^{H 2} / \lambda^{1 / 2}$ (without rest energy) for the Egger field (2.6) and the kinetic energy estimate from (2.4) with different trial functions.

(a) for $Z=80$ as a function of the hole diameter $r_{0}$ (in units of the $K$-shell radius, $1 / Z$ a.u.): $\psi_{t}$ for $\lambda=100(-)$ and $\lambda=10^{4}(-\cdot-\cdot-) ; \psi_{t}^{(2)}$ with $\tilde{\gamma}$ from (4.1) for $\lambda=100(-----)$ and $\lambda=10^{4}(\times)$. 
(b) for $\tilde{d}=3$ as a function of $\lambda$ for $\psi_{t}$ and $Z=80$ ( $\longrightarrow$ ), $Z=20(-\cdot-\cdot-)$, as well as for $\psi_{t}^{(3)}$ and $Z=80$ for $l=0.1(-----)$ and $l=1(\times)$.

D.H. Jakubassa-Amundsen, Mathematics Institute, University of Munich, ThereSienstr. 39, 80333 Munich, Germany

E-mail address: dj@mathematik.uni-muenchen.de 